\title{
Controlled short time large scale synthesis of magnetic cobalt nanoparticles on carbon nanotubes by flash annealing
}

\author{
Cite as: J. Appl. Phys. 127, 043902 (2020); doi: 10.1063/1.5131579 \\ Submitted: 15 October 2019 . Accepted: 13 December 2019 . \\ Published Online: 22 January 2020
}

\begin{abstract}
Aaron Mosey, ${ }^{7}$ (D) Lanping Yue, ${ }^{2}$ (D) Babu Gaire, ${ }^{7}$ (D) Jong Eun Ryu, ${ }^{3}$ (D) and Ruihua Cheng ${ }^{1, a)}$ (iD)
AFFILIATIONS

${ }^{7}$ Department of Physics, Indiana University_Purdue University Indianapolis, 402 N. Blackford St., Indianapolis, Indiana 46202, USA

${ }^{2}$ Nebraska Center for Materials and Nanoscience, University of Nebraska Lincoln, Nebraska 68588-0299, USA

${ }^{3}$ Department of Mechanical and Aerospace Engineering, North Carolina State University, Raleigh, North Carolina 27695, USA
\end{abstract}

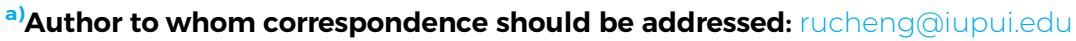

\begin{abstract}
Nanopatterned arrays of discrete cobalt nanostructures showing characteristic parameter-dependent sizes are formed from continuous thin films on a carbon nanotube substrate using millisecond pulsed intense UV light. The nanoparticles exhibit ferromagnetic behavior with magnetic remanence and coercivity depending on the particle size. The end-state particle size is shown to be a function of initial thin film thickness and excitation energy and is therefore tunable. The evolutionary process from continuous thin films to a discrete morphology is thermodynamically driven by the large surface energy difference between metastable thin films and the underlying carbon nanotube substrate. Evidence of the Danielson model of the dewetting process is observed. These arrays can find applications as platforms for the self-assembly of magnetically susceptible materials, such as iron or nickel nanostructures, into a conduction matrix for applications in energy extraction from a latent heat storage device.
\end{abstract}

Published under license by AIP Publishing. https://doi.org/10.1063/1.5131579

\section{INTRODUCTION}

Nanoscaled magnetic arrays with tunable end-state parameters, such as size and nearest-neighbor spacing, are key components to the development of next-generation microelectronic and nanoelectronic and mechanical devices. Such arrays find applications in medicine, security systems, environmental remediation, and data storage media, as the basis for building self-assembled nanostructures ${ }^{1-8}$ and a host of other operations.

Ubiquitous to nanotechnology, the utility of Carbon Nanotubes (CNTs) to both the scientific and engineering communities is at present well known. However, as we continue to push the envelope of technology, the ability to functionalize and harness objects which have both interesting transport and magnetic properties opens the door to viable platforms for spintronic and magnetoplasmonic device research. While CNTs with plasmon-active nanostructures such as Ag nanoparticles have shown applications in Raman signal amplification ${ }^{9}$ (Surface Enhanced Raman Spectroscopy or SERS), carbon nanotubes with magnetically active nanoparticles can provide a wonderful platform for exploration in the burgeoning field of nanomagnetoplasmonics. Indeed, the ability to combine and tailor tunable properties of light, magnetism, thermal response, and conductivity on the nanoscale, promises a wealth of highly applicable and fascinating phenomena.

One intriguing application is that of a self-assembled, nanoscaled conduction grid for the extraction of thermal energy in a Phase Change Material (PCM) based latent heat storage device. ${ }^{10}$ In this case, it may be desirable to keep an underlying conductive substrate intact as an integral part of the device. Also, processes to create a PCM thermal storage device for real world applications must facilitate large scale reel to reel production with low environmental impact and sufficient build times.

A Carbon Nanotube (CNT) substrate, in particular, a matrix comprised of CNTs, is an ideal substrate candidate for this purpose. Work to create nonmagnetic metallic nanoarrays grown on CNT substrates ${ }^{8,11,12}$ using a controlled dewetting process of thin films has been done by Ryu et al. by utilizing Intense Pulsed Light (IPL) solid state dewetting. This process takes place on a short (millisecond) time scale, does not damage the substrate or 
create harmful waste, and is able to be scaled up for high volume production needs.

Here, we report on a synthesis technique for producing a magnetic array by utilizing the low surface energy ${ }^{13}$ and cylindrical geometry of the CNT substrate to dewet metastable cobalt thin films into discrete magnetic particles. We explore the end-state parameter dependence on initial conditions and look for the evidence of a connection between beginning and end-state morphologies to a known model for dewetting.

Modern models that describe dewetting, or agglomeration, i.e., the evolution of a continuous body into a collection of discrete objects, may be traced back to the problem of a cylindrical liquid breaking up into constituent spheres in order to lower surface energy. This age-old problem was successfully described mathematically in the late 19th century. ${ }^{14}$ Subsequent works followed ${ }^{15-17}$ in more recent times, motivated by the thermal breakdown and ensuing failure of thin silicon on insulator films used in the microchip industry, culminating in the models put forth by Danielson. ${ }^{18}$ With the increasing need for the control of objects on the nanoscale, methods have been developed which harness this agglomeration process in order to create useful arrays of nanoparticles.

As-deposited planar thin films are usually metastable and thus subject to instabilities resulting in the dewetting of the thin film into a discrete final-state morphology. ${ }^{18,19}$ This thermodynamic process is driven by surface energy differentiation in the thin film, substrate, and environment of the system. When allowed to proceed into later stages, the evolution of the system yields a finalstate morphology of discrete particles that possess characteristic size and nearest-neighbor spacing. These attributes are parameter dependent and selective, relying upon initial conditions such as initial thin film thickness, excitation energy, time scale of exposure, and local substrate conditions.

\section{EXPERIMENT}

The synthesis of discrete arrays of magnetic nanostructures is achieved using the solid state dewetting technique via IPL excitation. The proper choice of a substrate to facilitate the agglomeration process is very important. An ideal substrate should readily dewet and remain thermally robust after flash annealing treatment. Substrates with low surface energy and good thermal conductivity, such as CNTs, are ideal choices.

Two types of substrates were used: an in-lab drop cast multiwalled CNT film and Buckypaper, a proprietary CNT matrix as supplied by Nanotech Labs, Inc. The in-lab CNT substrate was made by sonicating $5 \mathrm{mg}$ of multiwalled CNTs (as provided by Nanotech Labs, Inc.) into $20 \mathrm{ml}$ of acetone for $80 \mathrm{~min}$ for dispersion then drawing into a pipet and applying directly to a silicon wafer. As acetone evaporates, a dense, robust film of CNTs is left behind. The Buckypaper is comprised of multiwalled CNTs with diameters ranging from 5 to $80 \mathrm{~nm}$. For ease of transport, handling, and to facilitate imaging via scanning electron microscope (SEM), silver conductive paste was used to attach the Buckypaper to silicon wafers.

The thin films were grown using direct current magnetron sputtering under an inert (argon) atmosphere inside a high vacuum chamber with a sputtering source built by AJA International. The base pressure during the deposition was $10^{-7}$ Torr. Film growth rate parameters were calibrated using a single crystal quartz thickness monitor; the optimized growth rate was $1 \mathrm{~nm} / \mathrm{min}$. A series of samples were created which were masked, leaving a definitive step which was measured using a Bruker Axiovert atomic force microscope in a noncontact tapping mode. Multiple measurements were taken from these samples in order to verify the growth rate and ensure repeatability for nominal thicknesses.

Subsequent dewetting was achieved through IPL annealing using a xenon excitation source. The IPL source was a commercially available unit consisting of a xenon bulb attached to a capacitor bank with adjustable output energy of up to $50 \mathrm{~J} / \mathrm{cm}^{2}$. The parameters investigated include initial nominal thin film thickness, excitation energy, and the magnetic properties of the resultant nanoparticles. Particle size dependence and distribution characterization was done using a JEOL 7800F Field Emission Scanning Electron Microscope (FESEM). Magnetic properties were characterized using a Superconducting Quantum Interference Device (SQUID) Magnetometer. A series of samples with initial thin film nominal thicknesses of $5,10,15$, and $20 \mathrm{~nm}$ were fabricated and characterized.

\section{RESULTS AND DISCUSSION}

The low surface energy, high aspect ratio, and robust nature of a CNT substrate provide ideal conditions for solid state dewetting and subsequent nanoparticle agglomeration. In addition to chemical inertness and high thermal conductivity, CNT substrates possess desirable characteristics for subsequent device applications, such as being the conductive matrix in the self-assembled platforms for the fabrication of energy extraction devices for latent heat storage devices utilizing phase change materials.

When the entire system (substrates, thin films, and environment) is considered, the process can be understood thermodynamically $^{20}$ as an energy driven process. Because CNTs have very low surface energy compared with that of the metastable cobalt thin film, agglomeration proceeds in order to lower the total energy of the system as more substrate surface area is exposed. ${ }^{21}$

The model that best describes the agglomeration process for thin films on substrates in a planar configuration was developed by Danielson and consists of five distinct steps as follows: (I) critical void formation, (II) void edge thickening, (III) void edge breakdown, (IV) void finger formation and growth, and (V) island formation. Key processes include Rayleigh instabilities and capillary instabilities. ${ }^{22}$ An initiation of the process occurs at nucleation sites due to pre-existing voids, which may be in the form of strains at grain boundaries or imperfections on the substrate that protrude up into the film. If these voids are of a critical radius given by $R=2 h_{\circ} /(1-\cos \theta)$, where $h_{\circ}$ is the initial thin film thickness and $\theta$ is the contact angle between the film and the substrate, it will cause fingering and eventual breakup of the film into discrete islands.

As shown in Fig. 1, a discrete boundary is observed that separates a region with complete agglomeration, evidenced by populated nanoparticles, and a region where the thin film is intact. At the boundary of these two regions, evidence of the Danielson model and Rayleigh instabilities is clearly noticeable. In our case, the region for homogeneous light exposure on the surface of the substrate is smaller than the total surface area of the substrate. 
The sharp, discrete nature of the boundary indicates that a threshold energy is needed in order for dewetting to occur. This transition boundary would be difficult, if not impossible, to observe using traditional annealing methods such as furnace annealing. In addition, this may provide some insight into the nature of dewetting processes on the cylindrical geometry, and it should be compared to similar systems deposited on planar substrates.

Parameter dependence of end-state morphologies is apparent when considering particle size, nearest-neighbor spacing, and magnetic properties. End-state particle size dependence upon the excitation energy was established by characterizing a series of samples with $h_{\circ}=20 \mathrm{~nm}$ exposed to energies ranging from $10 \mathrm{~J} / \mathrm{cm}^{2}$ to $40 \mathrm{~J} / \mathrm{cm}^{2}$. We observed a systematic increase in the particle size as the energy increases. ${ }^{23}$ Higher excitation energy generates higher surface temperature. Mass flux diffusion, and hence agglomeration, is a temperature dependent process. The rate of the evolution process of agglomeration is accelerated as the mass diffusion rate from the void edges increases. Since all samples were exposed for the same time scale of $30 \mathrm{~ms}$, the samples exposed to higher energies progressed further into the agglomeration process, undergoing Ostwald ripening, ${ }^{24}$ in which discrete particles with larger mass subsume their smaller neighbors, should they fall within an effective interaction radius. The presence of larger particles under higher excitation energies, as well as the absence of smaller ones, points to the Ostwald ripening process.

Nanoparticles prepared with a range of initial thin film thicknesses also show a systematic dependence. Samples with initial thin film thicknesses of $5 \mathrm{~nm}, 10 \mathrm{~nm}, 15 \mathrm{~nm}$, and $20 \mathrm{~nm}$, all exposed to $50 \mathrm{~J} / \mathrm{cm}^{2}$ for $30 \mathrm{~ms}$, exhibited dependence of the initial thickness. As shown in Fig. 2, we observe progressively larger particles for thicker films. Agglomeration propagation has a strong dependence

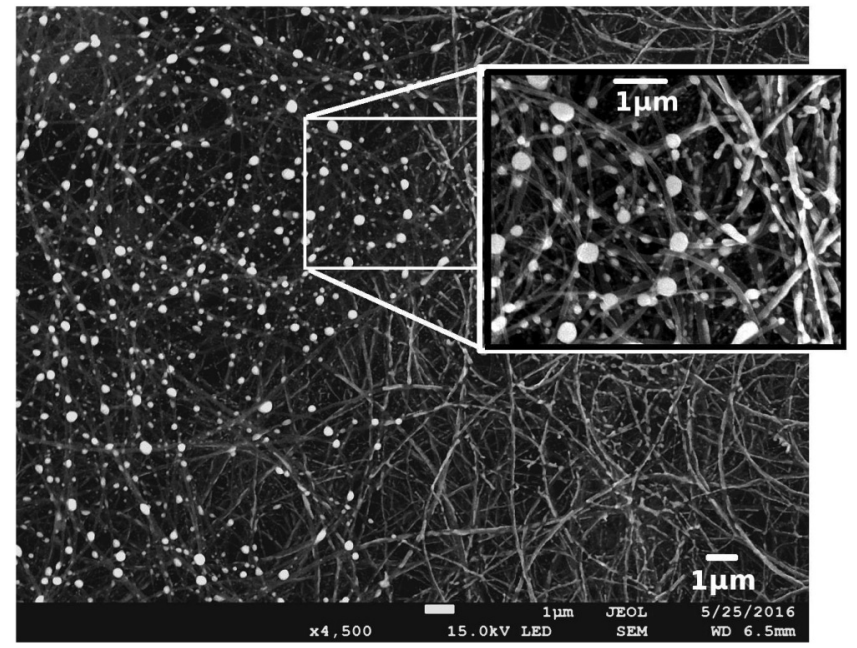

FIG. 1. A clear transition boundary (shown) indicates a threshold energy needed for solid state dewetting. $50 \mathrm{~J} / \mathrm{cm}^{2}$ excitation energy was used on a cobalt thin film of an initial nominal thickness $h_{\circ}=20 \mathrm{~nm}$. The inset shows the zoomed-in images of the indicated area. The scale bar denotes $1 \mu \mathrm{m}$ for both SEM images.

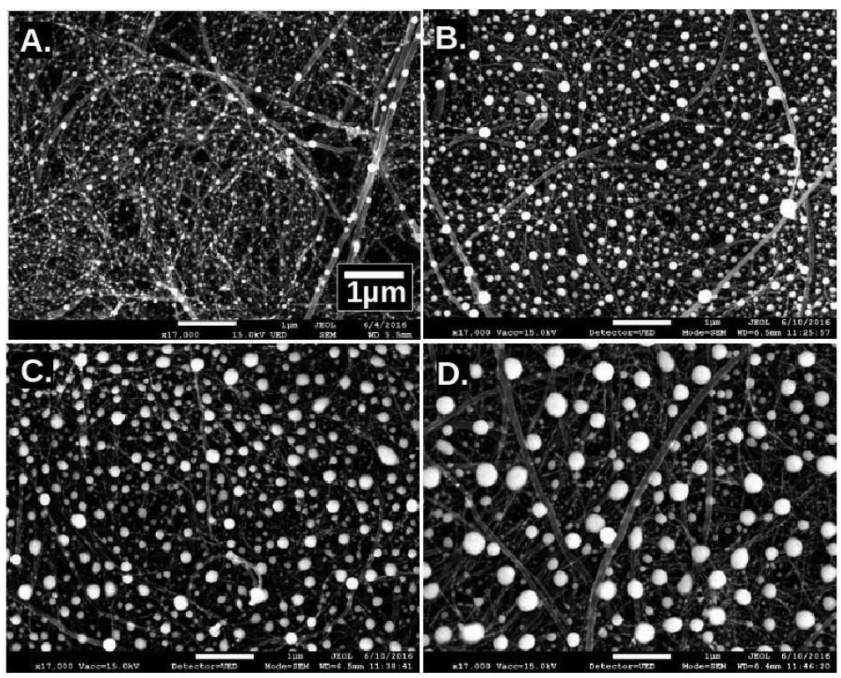

FIG. 2. Cobalt nanoparticles fabricated from an initial film thickness $h_{\circ}$ of (a) $5 \mathrm{~nm}$, (b) $10 \mathrm{~nm}$, (c) $15 \mathrm{~nm}$, (d) $20 \mathrm{~nm}$ with an excitation energy of $50 \mathrm{~J} / \mathrm{cm}^{2}$. Scale bar is $1 \mu \mathrm{m}$ for all SEM images.

upon critical void radius $R$, which is linearly proportional to the film thickness as in $R=\frac{2 h_{\circ}}{1-\cos \theta}$, where $h_{\circ}$ is the initial film thickness and $\theta$ is the contact angle, ${ }^{18,25}$ and the surface curvature of this edge goes as the inverse of its radius, which affects surface diffusion flux. Figure 3 shows this relationship to be a nearly linear reliance on $h_{\circ}$, with the error bars reflecting the polydispersity of the particle size. The theoretical models considered here are from a planar substrate paradigm. Our data show that this is still a good basic model even given the high aspect ratio and cylindrical geometry of a CNT based substrate. This reliance was observed for both the

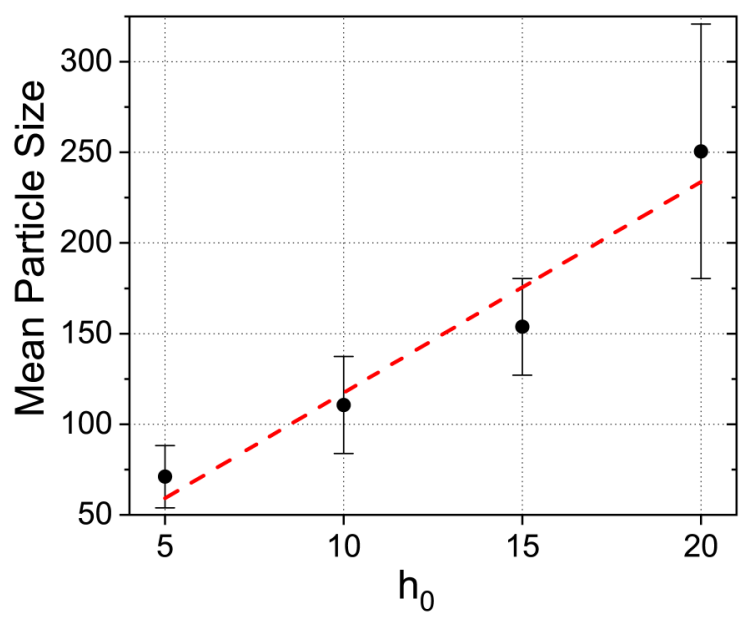

FIG. 3. Nanoparticle size as a function of initial thin film thickness $h_{0}$. 
in-lab made substrates as well as the Buckypaper; however, more uniform distribution of the particles was observed on the Buckypaper. The CNTs in the Buckypaper are more densely packed and more evenly distributed.

Magnetic measurements were carried out at room temperature using a SQUID magnetometer. Figure 4(a) shows normalized magnetic hysteresis loops of nanoparticle samples with different mean sizes, which are fabricated by manipulating the initial thin film thickness as discussed earlier. Those samples clearly show ferromagnetic properties indicted by noticeable magnetic remanence. We noticed that as the mean nanoparticle size increases, the magnetic coercivity $H_{c}$ is stable, then decreases dramatically as plotted in Fig. 4(b). This can be explained by magnetic domain structures.

When the mean particle size is small, the magnetic domain structure behaves as a single magnetic domain system; as the mean

(a)

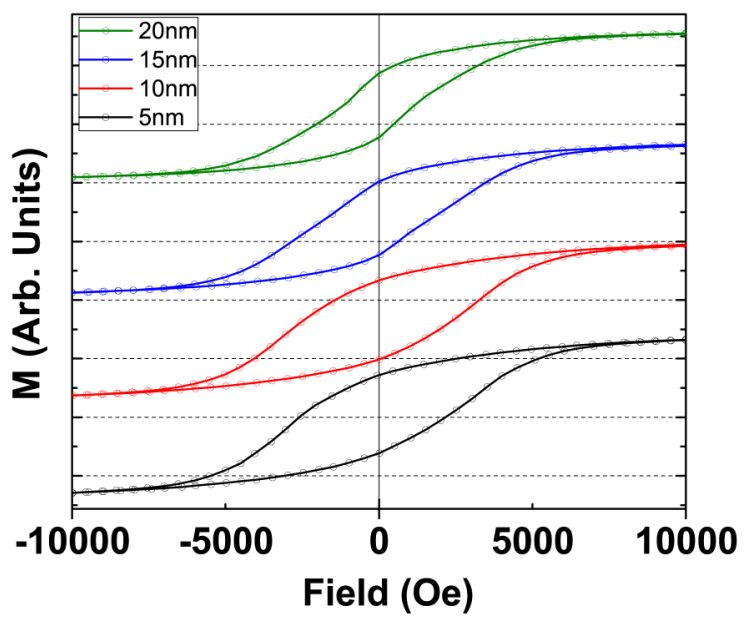

(b)

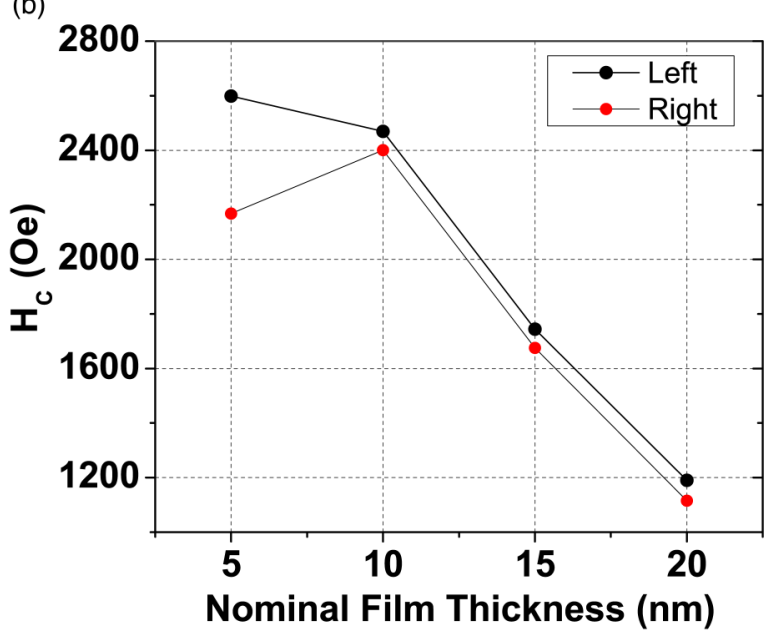

FIG. 4. (a) Magnetization curves measured at room temperature for nanoparticles created from different initial thin film thicknesses on the Buckypaper. (b) Coercivity as a function of nanoparticle size.

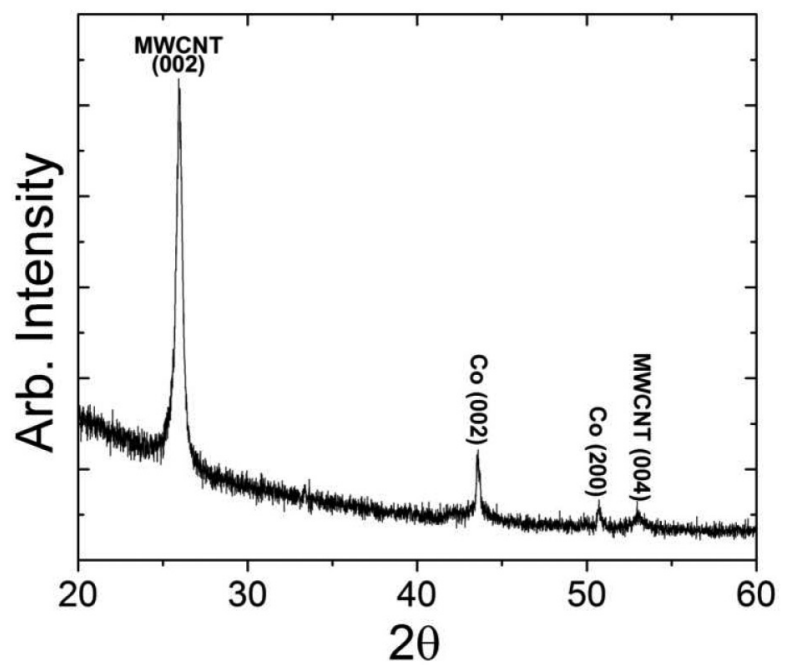

FIG. 5. XRD data from a nanoparticle sample. This sample was of $20 \mathrm{~nm}$ initial thin film thickness and that corresponds to a mean size of $110 \mathrm{~nm}$. We note the presence of peaks for cobalt.

size increases, the multidomain structure starts to dominate so the coercivity decreases. ${ }^{26,27}$ For small particles, the energy of the domain wall dominates hence single domain particles are stable below a critical size, and the magnetization reversal would occur with magnetization rotation as described by the Stoner-Wohlfarth model. For larger particles, the magnetic multidomain dominates and the magnetization reversal occurs through wall motion. The variation of the coercivity with the particle or crystallite size ${ }^{28}$ in the multidomain configuration is expressed as ${ }^{29} H_{c}=a+\frac{b}{D}$, where $a$ and $b$ are constants and $D$ is the diameter of the particle, indicating that as the particle diameter increases, the multidomain coercivity decreases. $^{30}$

This agrees with our data as summarized in Fig. 4(b). We also noticed that there is a slight difference in the coercivity fields for left and right sides, as shown by Fig. 4(b). This could be explained by exchange bias between ferromagnetic cobalt and a thin layer of antiferromagnetic cobalt oxide on the surface due to native oxidation. The composition of our samples is also confirmed by XRD data shown in Fig. 5. The peaks indicating cobalt and CNTs are noticeably present in the XRD data.

\section{CONCLUSIONS}

Arrays of ferromagnetic cobalt nanoparticles with a tunable size were formed on low surface energy carbon nanotube substrates using intense pulse light solid state flash annealing. The end-state morphology including the particle size was shown to be dependent upon initial conditions and energy parameters. A region of partial dewetting transitioning to continuous thin films, indicating an energy threshold for dewetting, was observed. This region shows evidence for capillary instabilities and Rayleigh instabilities, giving evidence that the Danielson model for dewetting (or particle growth) 
fits this system. Magnetic analysis by SQUID magnetometry indicates that the magnetic coercivity of the ferromagnetic nanoparticles depends upon the particle size. As the mean particle size increases, the coercivity decreases and the magnetic structure shows a transition from single domain to multidomain.

\section{ACKNOWLEDGMENTS}

The research was performed in part in the Nebraska Nanoscale Facility: National Nanotechnology Coordinated Infrastructure and the Nebraska Center for Materials and Nanoscience, which are supported by the National Science Foundation (NSF) under Award No. ECCS 1542182 and the Nebraska Research Initiative.

\section{REFERENCES}

${ }^{1}$ S. Mornet, S. Vasseur, F. Grasset, P. Veverka, G. Goglio, A. Demourgues, J. Portier, E. Pollert, and E. Duguet, "Magnetic nanoparticle design for medical applications,” Prog. Solid State Chem. 34, 237-247 (2006).

${ }^{2}$ D. W. Elliott and W.-X. Zhang, "Field assessment of nanoscale bimetallic particles for groundwater treatment," Environ. Sci. Technol. 35, 4922-4926 (2001).

${ }^{3}$ D. Weller and M. F. Doerner, "Extremely high density longitudinal recording media," Annu. Rev. Mater. Sci. 30, 611-644 (2000).

${ }^{4}$ C. M. Hangarter and N. V. Myung, "Magnetic alignment of nanowires," Chem. Mater. 17, 1320-1324 (2005).

${ }^{\mathbf{5}}$ L. He, M. S. Wang, J. P. Ge, and Y. D. Yin, "Magnetic assembly route to colloidal responsive photonic nanostructures," Acc. Chem. Res. 45, 1431-1440 (2012).

${ }^{6}$ M. Tanase, D. M. Silevitch, A. Hultgren, L. A. Bauer, P. C. Searson, G. J. Meyer, and D. H. Reich, "Magnetic trapping and self-assembly of multicomponent nanowires," J. Appl. Phys. 91, 8549-8551 (2002).

${ }^{7}$ I. Kavre, G. Kostevc, S. Kralj, A. Vilfan, and D. Babič, "Fabrication of magnetoresponsive microgears based on magnetic nanoparticle embedded PDMS," RSC Adv. 4, 38316-38322 (2014).

${ }^{8}$ J. Ryu, K. Kim, H.-S. Kim, H. T. Hahn, and D. Lashmore, "Intense pulsed light induced platinum-gold alloy formation on carbon nanotubes for non-enzymatic glucose detection,” Biosens. Bioelectron. 26, 602-607 (2010).

${ }^{9}$ X. Zhang, J. Zhang, J. Quan, N. Wang, and Y. Zhu, "Surface-enhanced Raman scattering activities of carbon nanotubes decorated with silver nanoparticles," Analyst 141, 5527-5534 (2016).

${ }^{10}$ J. Su, I. Mirzaee, F. Gao, X. Liu, M. Charmchi, Z. Gu, and H. Sun, "Magnetically assembling nanoscale metal network into phase change materialPercolation threshold reduction in paraffin using magnetically assembly of nanowires," J. Nanotechnol. Eng. Med. 5, 031005 (2014).
${ }^{11}$ J. Ryu, H. S. Kim, H. T. Hahn, and D. Lashmore, "Carbon nanotubes with platinum nano-islands as glucose biofuel cell electrodes," Biosens. Bioelectron. 25, 1603-1608 (2010).

${ }^{12}$ A. P. U. Kulkarni, "Au nanoparticle assembly on CNTs using flash induced solid-state dewetting," Ph.D. thesis (Purdue University, 2015).

${ }^{13}$ S. Nuriel, L. Liu, A. H. Barber, and H. D. Wagner, "Direct measurement of multiwall nanotube surface tension,” Chem. Phys. Lett. 404, 263-266 (2005).

${ }^{14}$ L. Rayleigh, "On the instability of jets," Proc. Lond. Math. Soc. s1-s10, 4-13 (1878).

${ }^{15}$ W. W. Mullins, "Flattening of a nearly plane solid surface due to capillarity," J. Appl. Phys. 30, 77-83 (1959).

${ }^{16}$ F. A. Nichols and W. W. Mullins, "Morphological changes of a surface of revolution due to capillarity-induced surface diffusion," J. Appl. Phys. 36, 1826-1835 (1965).

${ }^{17}$ G. Reiter, "Dewetting of thin polymer films," Phys. Rev. Lett. 68, 75-78 (1992).

${ }^{18}$ D. T. Danielson, D. K. Sparacin, J. Michel, and L. C. Kimerling, "Surface-energy-driven dewetting theory of silicon-on-insulator agglomeration," J. Appl. Phys. 100, 083507 (2006).

${ }^{19}$ C. V. Thompson, "Solid-state dewetting of thin films," Annu. Rev. Mater. Res. 42, 399-434 (2012).

${ }^{20}$ D. J. Srolovitz and M. G. Goldiner, "The thermodynamics and kinetics of film agglomeration," JOM 47, 31 (1995).

${ }^{21}$ D. J. Srolovitz and S. A. Safran, "Capillary instabilities in thin, polycrystalline films," MRS Proc. 47, 23-28 (1985).

${ }^{22}$ D. J. Srolovitz and S. A. Safran, "Capillary instabilities in thin films. I. Energetics,” J. Appl. Phys. 60, 247-254 (1986).

${ }^{23}$ A. Mosey, B. Gaire, J. Kim, J. Ryu, and R. Cheng, "Tunable cobalt nanoparticle synthesis by intense pulse flash annealing," AIP Adv. 7, 056308 (2017).

${ }^{24}$ I. Beszeda, E. G. Gontier-Moya, and W. Imre, "Surface Ostwald-ripening and evaporation of gold beaded films on sapphire," Appl. Phys. A Mater. Sci. Process. 81, 673-677 (2005).

${ }^{25}$ J. H. Yao, K. R. Elder, H. Guo, and M. Grant, "Theory and simulation of Ostwald ripening," Phys. Rev. B 47, 14110-14125 (1993).

${ }^{26}$ D. J. Srolovitz and S. A. Safran, "Capillary instabilities in thin films. 2: Kinetics,” J. Appl. Phys. 247, 255-260 (1986).

${ }^{27}$ J. S. Lee, J. M. Cha, H. Y. Yoon, J. K. Lee, and Y. K. Kim, "Magnetic multigranule nanoclusters: A model system that exhibits universal size effect of magnetic coercivity," Sci. Rep. 5, 1-7 (2015).

${ }^{28} \mathrm{Q}$. Li, C. W. Kartikowati, S. Horie, T. Ogi, T. Iwaki, and K. Okuyama, "Correlation between particle size/domain structure and magnetic properties of highly crystalline $\mathrm{Fe}_{3} \mathrm{O}_{4}$ nanoparticles," Sci. Rep. 7, 1-4 (2017).

${ }^{29}$ A. E. Berkowitz and W. J. Schuele, "Magnetic properties of some ferrite micropowders," J. Appl. Phys. 30, S134-S135 (1959).

${ }^{30}$ S. M. Ramay, M. Saleem, S. Atiq, S. A. Siddiqi, S. Naseem, and M. Sabieh Anwar, "Influence of temperature on structural and magnetic properties of $\mathrm{Co}_{0.5} \mathrm{Mn}_{0.5} \mathrm{Fe}_{2} \mathrm{O}_{4}$ ferrites,” Bull. Mater. Sci. 34, 1415-1419 (2011). 